Original article

\title{
Synthesis and characterization of a methacrylate monomer with a thiohydantoin structure
} \author{
and Hiroyasu Koizumi ${ }^{6,7)}$ \\ Department of Fixed Prosthodontics, Nihon University School of Dentistry, Tokyo, Japan \\ 2) Division of Advanced Dental Treatment, Dental Research Center, Nihon University School of Dentistry, Tokyo, Japan \\ ${ }^{3}$ Private Scholar, Oamishirasato, Japan \\ R\&D Center, Mitsui Chemicals Inc., Sodegaura, Japa \\ R\&D Department, Sun Medical Co., Ltd., Moriyama, Japan \\ Department of Dental Materials, Nihon University School of Dentistry, Tokyo, Japan \\ Division of Biomaterials Science, Dental Research Center, Nihon University School of Dentistry, Tokyo, Japan
}

Hideo Matsumura ${ }^{1,2)}$, Katsunori Kojima ${ }^{3)}$, Shigeru Mio $^{4}$, Takashi Yamamoto ${ }^{5)}$, Akihisa Kodaira ${ }^{1,2)}$,

\begin{abstract}
The purpose of the currentstudy was to synthesize a methacrylate monomer with a thiohydantoin structure. In a flask, (2-thiohydantoin-4-yl) propionic acid, 10-hyroxydecyl methacrylate, 4-dimethylaminopyridine, and tetrahydrofuran (THF) were placed in a nitrogen atmosphere. A THF solution of $N, N^{\prime}$-dicyclohexylcarbodiimide was added and stirred at room temperature. Ethyl acetate and $n$-hexane were then added to the reaction mixture. The reacted slurry was purified by chromatography with silica gel and hexane-ethyl acetate gradient elution solvent. The structure of the compound was assigned with proton nuclear magnetic resonance spectrum analysis, infrared spectroscopy, and high-resolution mass spectral analysis. The colorless viscous liquid obtained in yield of $49 \%$ was characterized as 10-methacryloyloxydecyl-(2-thiohydantoin-4-yl)propionate (MDTHP) or 10-((3-(5-oxo-2-thioxysoimidazolidin-4-yl)propanoyl)oxy)decyl methacrylate (2-THPDM). The MDTHP was soluble to acetone, isopropanol, and methyl methacrylate up to $2.6 \%$ or more. This compound could be potentially applicable as a functional monomer for bonding metals and alloys.
\end{abstract}

Keywords; adhesive, bonding, noble metal alloy, thiol, thione

\section{Introduction}

Vinyl and methacrylic monomers with mercapto, thioxo, or sulfide groups have been used for priming copper and noble metals. A triazine thiol, 6-(4-vinylbenzyl- $n$-propyl) amino-1,3,5-triazine-2,4-dithiol (VBATDT or VTD) [1], was used as a coating agent for copper plating. A thiophosphate, 10-methacryloyloxydecyl dihydrogen thiophosphate (M10PS, MDTP) (Kojima et al., J J Dent Mater 6 Spec 10, 112, 1987) [2]; a disulfide, methacryloyloxydecyl 6,8-dithiooctanoate (MDDT) [3,4]; and a thiouracil, 6-methacryloyloxyhexyl 2-thiouracil-5-carboxylate (MTU-6) [5], have been reported as functional methacrylates. These monomers are dissolved in solvents and are being used as single liquid priming agents capable of bonding dental noble metal alloys [6-12].

Thiohydantoin is one of the five-membered heterocyclic compounds with thione structure. However, characteristics of thiohydantoin derivatives with polymerizable functional groups have not been reported. This report describes the synthesis of a thiohydantoin compound with a methacryloyloxydecyl group.

\section{Materials and Methods}

\section{Reagents}

Reagents, $N, N^{\prime}$-dicyclohexylcarbodiimide (DCC) and 4-dimethylaminopyridine (DMAP), were commercially obtained and purified by recrystallization. Ethyl acetate, $n$-hexane, tetrahydrofuran (THF), and

Correspondence to Dr. Hideo Matsumura, Department of Fixed Prosthodontics, Nihon University School of Dentistry, 1-8-13 Kanda-Surugadai, Chiyoda-ku, Tokyo 101-8310, Japan

E-mail: matsumura.hideo@nihon-u.ac.jp

J-STAGE Advance Publication: May 15, 2020

doi.org/10.2334/josnusd.19-0005

DN/JST.JSTAGE/josnusd/19-0005 1,10-decane diol were purified by distillation. Reagent grade 2,6-di-tertbutyl-4-methylphenol, i.e. 3,5-di-tert-butyl-4-hydroxytoluene (BHT); acetone; isopropanol; and methyl methacrylate (MMA) were used without further purification. VBATDT was synthesized according to the method described by Mori and Nakamura [1].

\section{0-hyroxydecyl methacrylate (HDMA)}

Methacryloyl chloride was synthesized by reacting glacial methacrylic acid and thionyl chloride in the presence of cuprous chloride [13]. Methacryloyl chloride was then reacted with 1,10-decanediol with equivalent molar ratio in THF. Pyridine was used as the de-hydrochloric acid agent. The mixture of HDMA and 1,10-decanediol dimethacrylate was separated through column chromatography [14].

\section{(2-thiohydantoin-4-yl)propionic acid (THPA)}

A thiohydantoin derivative, THPA, was synthesized according to the method described by Szabo and Karabinos [15] and Duggan et al. [16].

Synthesis and characterization of a methacrylate with thiohydantoin structure

In a $300-\mathrm{mL}$ four-necked flask, $4.45 \mathrm{~g}(23.6 \mathrm{mmol})$ THPA, $6.88 \mathrm{~g}(28.4$ mmol) HDMA, $0.58 \mathrm{~g}$ (4.73 mmol) DMAP, $7.0 \mathrm{mg}$ BHT, and $90 \mathrm{~mL}$ THF were placed in a nitrogen atmosphere. A solution of $5.82 \mathrm{~g}(24.8 \mathrm{mmol})$ DCC in $30 \mathrm{~mL}$ THF was dropped from a funnel and stirred for $4.5 \mathrm{~h}$ at room temperature. Next, $150 \mathrm{~mL}$ ethyl acetate and $30 \mathrm{~mL} n$-hexane were added to the reaction mixture and stirred for $10 \mathrm{~min}$.

The reacted whitish slurry was filtrated through a pad of Hyflo Super Cel (Fujifilm Wako Pure Chemical Corp., Osaka, Japan), and the filtrate was condensed under reduced pressure. The oily product was purified twice by chromatography with $100 \mathrm{~g}$ silica gel (SNAP KP-Sil Cartridge, Biotage Japan Ltd., Tokyo, Japan) and hexane-ethyl acetate (5-100\%) gradient elution solvent.

The structure of the compound was assigned with proton nuclear magnetic resonance $\left({ }^{1} \mathrm{H}-\mathrm{NMR}\right)$ spectrum analysis $(270 \mathrm{MHz}$, JEOL Excalibur 270, JEOL Ltd., Akishima, Japan) in $\mathrm{CDCl}_{3}$. Chemical shifts $(\delta)$ were expressed in parts per million and are internally referenced with tetramethylsilane. Infrared (IR) spectroscopy (Spectrum Tow N FT-IR, PerkinElmer Japan Co., Ltd., Yokohama, Japan) was used to analyze the functional groups of the synthesized compound. The exact mass of the synthesized compound was determined by high-resolution mass spectral (HRMS) analysis (Acquity UPLC system/Xevo G2 QT Tof, Nihon Waters K.K., Tokyo, Japan).

\section{Solubility of monomers}

The solubility of the synthesized monomer in the current project and VBATDT [1] was evaluated. The solvents used were acetone, isopropanol, and MMA. Monomers were separately added to the three solvents and magnetically stirred for $30 \mathrm{~min}$. The resultant transparent solution was judged as a solution within the soluble wt $\%$ concentration of each monomer. 


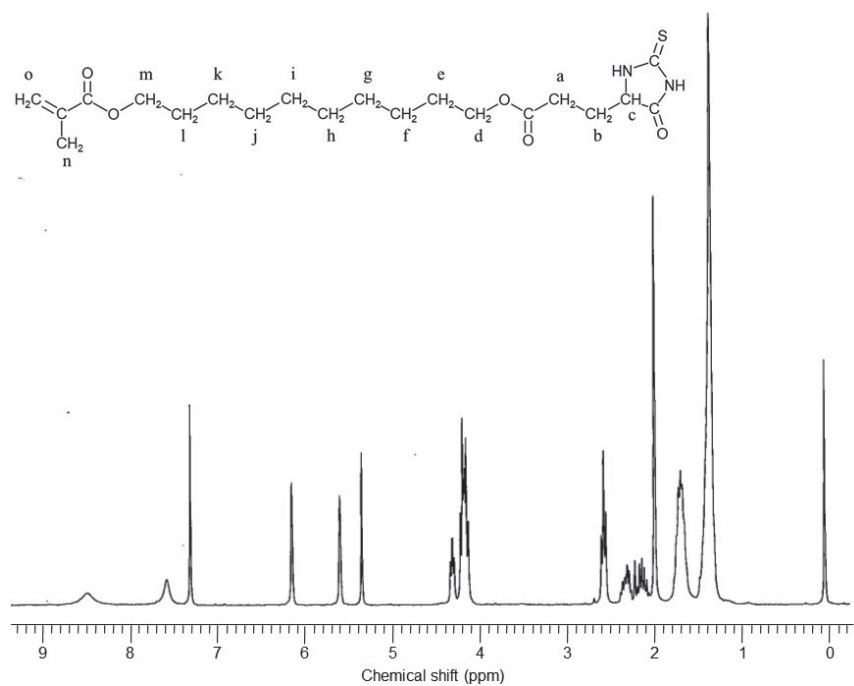

Fig. 1 'H-NMR spectrum analysis result for MDTHP

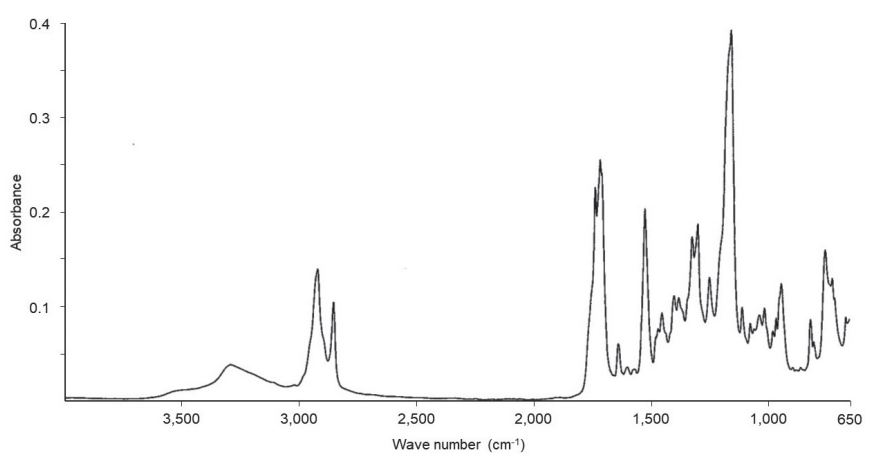

Fig. 2 Infrared (IR) spectrum analysis result for MDTHP

Table 1 Assignment of the monomer synthesized

\begin{tabular}{|c|c|c|c|c|}
\hline \multicolumn{5}{|l|}{${ }^{1} \mathrm{H}-\mathrm{NMR}$} \\
\hline Chemical sift (ppm) & Integral value & Assignment* & & \\
\hline 8.45 & $1 \mathrm{H}$ & $\mathrm{N} H$ & singlet, broad & \\
\hline 7.53 & $1 \mathrm{H}$ & $\mathrm{N} H$ & singlet, broad & \\
\hline 6.10 & $1 \mathrm{H}$ & Но & multiplet & \\
\hline 5.55 & $1 \mathrm{H}$ & Но & multiplet & \\
\hline 4.26 & $1 \mathrm{H}$ & $\mathrm{Hc}$ & doublet of doublet & $\mathrm{J}=6.9,4.9 \mathrm{~Hz}$ \\
\hline 4.14 & $2 \mathrm{H}$ & $\mathrm{Hm}$ or $\mathrm{Hd}$ & triplet & $\mathrm{J}=6.9 \mathrm{~Hz}$ \\
\hline 4.10 & $2 \mathrm{H}$ & $\mathrm{Hd}$ or $\mathrm{Hm}$ & triplet & $\mathrm{J}=6.9 \mathrm{~Hz}$ \\
\hline 2.53 & $2 \mathrm{H}$ & $\mathrm{Ha}$ & triplet & $\mathrm{J}=6.9 \mathrm{~Hz}$ \\
\hline $2.34-2.21$ & $1 \mathrm{H}$ & $\mathrm{Hb}$ & multiplet & \\
\hline $2.17-2.01$ & $1 \mathrm{H}$ & $\mathrm{Hb}$ & multiplet & \\
\hline 1.95 & $3 \mathrm{H}$ & $\mathrm{Hn}$ & multiplet & \\
\hline $1.72-1.60$ & $4 \mathrm{H}$ & $\mathrm{H}(\mathrm{e}, \mathrm{l})$ & multiplet & \\
\hline $1.43-1.26$ & $12 \mathrm{H}$ & $H(f, g, h, i, j, k)$ & multiplet & \\
\hline IR (ATR) & & & HRMS (ESI) $\mathrm{C}_{20} \mathrm{H}_{3}$ & \\
\hline Wave number $\left(\mathrm{cm}^{-1}\right)$ & Assignment & & Calcuated & Found \\
\hline 3,293 & $\mathrm{C}(=\mathrm{O}) \mathrm{NH}$ & & 413.211 & 413.2111 \\
\hline 2,919 & $\mathrm{CH}$ & & & \\
\hline 2,852 & $\mathrm{CH}$ & & & \\
\hline 1,734 & $\mathrm{C}=\mathrm{O}$ & & & \\
\hline 1,713 & $\mathrm{C}(=\mathrm{O}) \mathrm{O}$ & & & \\
\hline 1,705 & $\mathrm{NHC}=\mathrm{O}$ & & & \\
\hline
\end{tabular}

*Proton assignment in Fig. 1

Table 2 Solubility of MDTHP and VBATDT to solvents

\begin{tabular}{lccc}
\hline & $2.6 \%$ MDTHP & $0.65 \%$ MDTHP & $2.6 \%$ VBATDT \\
\hline Acetone & + & + & + \\
Isopropanol & + & + & + \\
MMA & + & + & - \\
\hline
\end{tabular}

+ , soluble;,- insoluble

\section{Results}

The colorless viscous liquid obtained from the condensation of 23.6 mmol THPA and 28.4 mmol HDMA was $4.79 \mathrm{~g}$ in yield of $49 \%$. Figures 1,2 and Table 1 summarize the results of analysis of the synthesized compound. From the results of ${ }^{1} \mathrm{H}-\mathrm{NMR}$ spectrum analysis, IR spectroscopy, and HRMS analysis, the compound was characterized as 10-methacryloyloxydecyl-(2-thiohydantoin-4-yl)propionate (MDTHP) or 10-((3-(5-oxo-2-thioxysoimidazolidin-4-yl)propanoyl)oxy)decyl methacrylate (2-THPDM).

Table 2 demonstrates a comparison of solubility to organic solvents of 2-THPDM and VBATDT. Both monomers were soluble to acetone up to $2.6 \%$. However, solubility of VBATDT to MMA and isopropanol was less than $0.65 \%$.

\section{Discussion}

Several thiols, thiones, and sulfides have been used for the surface modification of copper and noble metals. Because of the chain transfer reactions in polymerizable solvents or monomer liquids, it has been difficult to synthesize monomers with both mercapto and methacryloyl groups. The VBATDT reported by Mori and Nakamura [1] exhibited a thione structure after recrystallization from diethyl ether and $n$-hexane. However, a thioxo group of the VBATDT disappeared after absorption to copper or noble metals possibly because this group tautomerized into a mercapto group to form a bond between sulfur and metal elements. Based on the findings, organic sulfur compounds with methacryloyl groups have been synthesized. Among them, MTU-6 and MDDT have effectively enhanced the bonding performance of noble metals and alloys [4,7-12].

Thiohydantoin is a five-numbered cyclic compound with a thioxo 
group. In order to improve hydrophobicity, 10-hydroxydecyl methacrylate was used for the esterification of THPA. The synthesized monomer was not able to distill or recrystallize owing to the number of methylene groups incorporated into the monomer structure. The monomer was therefore purified with solvent extraction followed by column chromatography. As shown in Table 1 and Fig. 1, the monomer was purified with a yield of $49 \%$. However, care was taken during the elimination of the solvent to avoid polymerization of the purified monomer. This oily compound was soluble in acetone, isopropanol, and MMA up to $2.6 \%$; this concentration was sufficient for it to be used as a priming agent, with desirable characteristics compared with VBATDT.

Although the bonding performance of this compound to metals and alloys has not been evaluated, this monomer may be potentially applicable as a functional adhesive monomer for bonding between acrylic resin-based materials and noble metals and alloys.

\section{Acknowledgments}

This study was supported in part by a grant from Sun Medical Co., Ltd. (2017 and 2018).

\section{Conflict of interest}

Hideo Matsumura, Takashi Yamamoto, and Shigeru Mio applied for a patent: Sulfur-containing polymerizable monomer and use of thereof. International Laid-Open Patent Publication, No. WO2020/032268 A1, Sun Medical Co., Ltd., February 13, 2020.

\section{References}

1. Mori K, Nakamura Y (1983) Study on triazine thiols. V. polymerization of 6-(4-vinylbenzyl propyl)amino-1,3,5-triazine-2,4-dithiol on copper plates and their corrosion resistance. J Polym Sci Polym Lett Ed 21, 889-895.
2. Kojima K, Kadoma Y, Imai Y (1989) Japanese unexamined patent application publication No. Hei 1-138282.

3. Kojima K, Kadoma Y, Ikemura K (1997) (Meth)acrylic ester derivatives. United States Patent No. 5,670,657.

4. Ikemura K, Kojima K, Endo T, Kadoma Y (2011) Synthesis of novel acryloyloxyalkyl and methacryloyloxyalkyl 6,8-dithiooctanoates and evaluation of their bonding performances to precious metals and alloys. Dent Mater J 30, 827-836.

5. Kimura M, Aizawa M (1998) Japanese unexamined patent application publication No. Hei 10-1473.

6. Atsuta M, Matsumura H, Tanaka T (1992) Bonding fixed prosthodontic composite resin and precious metal alloys with the use of a vinyl-thiol primer and an adhesive opaque resin. J Prosthet Dent 67, 296-300.

7. Matsumura H, Shimoe S, Nagano K, Atsuta M (1999) Effect of noble metal conditioner on bonding between prosthetic composite material and silver-palladium-copper-gold alloy. J Prosthet Dent 81,710-714.

8. Minami H, Murahara S, Suzuki S, Tanaka T (2011) Effects of metal primers on the bonding of an adhesive resin cement to noble metal ceramic alloys after thermal cycling. J Prosthet Dent 106, 378-385.

9. Yamashita M, Koizumi H, Ishii T, Nakayama D, Oba Y, Matsumura H (2013) Adhesive performance of silver-palladium-copper-gold alloy and component metals bonded with organic sulfur-based priming agents and a tri-n-butylborane initiated luting material. Acta Odontol Scand 71, 196-204.

10. Hiraba H, Koizumi H, Nogawa H, Kodaira A, Okamura K, Matsumura H (2017) Trace of organic sulfur compounds detected from debonded interface between transparent acrylic resin and gold alloy. J Oral Sci 59, 511-517.

11. Imamura N, Kawaguchi T, Shimizu H, Takahashi Y (2018) Effect of three metal priming agents on the bond strength of adhesive resin cement to $\mathrm{Ag}-\mathrm{Zn}$-Sn-In alloy and component metals. Dent Mater J 37, 301-307.

12. Hiraba H, Nogawa H, Koizumi H, Kodaira A, Akahane S (2019) Effect of multi-purpose primers on the bond durability between tri-n-butylborane initiated resin and gold alloy. J Prosthodont Res 63, 95-99.

13. Lal J, Green R (1955) The preparation of some esters of methacrylic acid. J Org Chem 20, 1030-1033.

14. Inaishi K, Nakaya T, Imoto M (1975) Synthesis and polymerization of 10-[bis(benzyloxy) phosphoryloxy]decyl methacrylate. Makromol Chem 176, 2473-2478.

15. Szabo JL, Karabinos JV (1944) The dehydration of hydantoin-5-propionic acid. J Am Chem Soc 66, 650-651.

16. Duggan BM, Laslett RL, Wilshire JFK (1996) Studies in thiohydantoin chemistry. I. Some aspects of the Schlack-Kumpf reaction. Aust J Chem 49, 541-550. 